\title{
PSIR: a novel phase-wise diffusion model for lockdown analysis of COVID-19 pandemic in India
}

\author{
Adwitiya $\operatorname{Sinha}^{1}$ (D)
}

Received: 26 February 2021/Revised: 31 August 2021/Accepted: 22 October 2021 / Published online: 28 October 2021

(C) The Author(s) under exclusive licence to The Society for Reliability Engineering, Quality and Operations Management (SREQOM), India and The Division of Operation and Maintenance, Lulea University of Technology, Sweden 2021

\begin{abstract}
The coronavirus pandemic has damaged economies and hampered human lives with fatalities and socioeconomic losses. The emergency intervention measures imposed in several nations during the primary stage of the contagion had stalled the deadly virus from spreading explosively. One such preventive measures include the nation-wide lockdown process that became a significant step towards mitigation of COVID-19. In the proposed work, a diffusion-based system is proposed to analyze the impact of lockdown in India. The COVID spread data was obtained from the aggregated situation reports made available by the World Health Organization. The infection and recovery rate, being phase-dependent parameters, are computed from lockdown analysis. The reproduction number of coronavirus is also estimated for each phase, which was found to decrease consistently from initial to final phase during the lockdown. Further, the Phase-wise Susceptible-Infected-Recovered network diffusion system is proposed to model the phase-wise evolution of coronavirus spread in India is assessed. The proposed system simulated the lockdown that was imposed in India into five phases for an initial duration of 98 days. The infection and recovery rate during lockdown were used to be an important parameter in diffusion to measure the spreading potential of the deadly virus. Finally, the research highlights the benefits of re-imposing lockdown in India for
\end{abstract}

Adwitiya Sinha

mailtoadwitiya@gmail.com

1 Department of Computer Science \& Engineering and Information Technology, Jaypee Institute of Information Technology, Sector-62, Noida, Uttar Pradesh, India controlling the devastating COVID-19 situations, based on pre and post lockdown situation.

Keywords Coronavirus pandemic - Lockdown phase . Unlocking phase - Performance analysis - Recovery rate . Network diffusion-based system - Pandemic curve .

COVID-19 - Sars-CoV-2

\section{Introduction}

Pandemics have ravaged human civilization with its venomous contagion impact on a massive scale. A pandemic is a rapid outbreak of a contagious disease affecting people worldwide. It results in wide-spread morbidity and mortality causing the situation of a global health emergency. A pandemic is caused by an infectious agent that results in broad-spectrum mortality. The infectious agent spreads from person-to-person, thereby diffusing widely throughout communities and claiming lives. In December 2019, Wuhan was hit by an epidemic from Severe Acute Respiratory Syndrome Coronavirus 2 (SARS-CoV-2), also known as Coronavirus-19 (COVID-19). The widespread symptoms of coronavirus include fever, cough, chest pain, headaches, loss of taste, and smell. The epidemiologic characteristics of the coronavirus soon became an international concern and were declared a deadly pandemic on March 11, 2020, by World Health Organization (WHO) (Cucinotta and Vanelli 2020). The people with weak immunity, multiple ailments, and poor health standards become aggressively vulnerable towards developing severe COVID-19 symptoms, especially recurring respiratory infections. 
The first case of coronavirus in India was reported on 30th January 2020, and the first death casualty was recorded on 11th March, who died of COVID-19. This marked the rise of the pandemic in the country that engulfed major metropolitans and industrial hubs. Several measures were initiated in India to control the worsening situation as existing medication and treatment procedures were found ineffective. One of the major steps taken was the phasewise lockdown for slowing down the pace of community spread. The effects of lockdown in India are manifold and wide-reaching. Moreover, the lockdown was considered a significant intervention in the mitigation and management of SARS-CoV-2. The lockdown strategy ensured:

- Restricted mobility for the susceptible population

- Social distancing, especially for ones engaged in essential services

- Isolation of suspected cases of coronavirus

- Quarantined treatment of the confirmed cases

The goal of implementing lockdown was solely to curb off the pandemic by reducing the reproducibility of the virus. The research is focused on analyzing the change in the rate of spread of the COVID-19 pandemic using proposed Phase-wise Susceptible-Infected-Recovered (PSIR) diffusion model through the consecutive five major phases of lockdown in India. The experimentation shows a drop in reproduction number of coronavirus, $R_{0}$ after each lockdown phase from 25th March to 30th June 2020. Some of the deciding parameters taken for examination of corona cases include recovery rate, death rate, infection rate, death-to-active ratio, and death-to-recovery ratio.

\section{Background study}

The large effectivity and transmissibility of COVID-19 spread currently brought the entire humanity of the world under risk. This virus, also known as novel coronavirus, is an inflammatory disease attacking the respiratory organs with manifestations of cold, cough fever, and breathing troubles. Due to its high contagion nature, WHO declared it as a global public health emergency and circulated guidelines and released worldwide help portals to create awareness and maintain basic health amenities. After the coronavirus outbreak in China, the cases of COVID-19 begun to emerge in Italy by the end of January 2020. Severe restrictions started being imposed, like sudden lockdown and quarantined treatment in the country. The COVID-19 spread triggered physiological as well as psychological ailments, including depression and post-traumatic stress disorder (PTSD) (Castelli et al. 2020). There are several computational methods, including intelligent data analysis and informatics for identifying disease spread pattern, diffusion analysis, and decision making (Gurevich and Zhuravlev 2014; Vissia et al. 2020; Veera Krishna 2020). Moreover, case studies over various countries across the world started to be conducted for analysing the growth phenomena of the pandemic (Sebhatu et al. 2020; Sinha and Rathi 2021; Zemtsov and Baburin 2020).

In a recent work, the authors have explored data-driven forecasting methodology based on long short-term memory (LSTM) for the estimation of positive, recovered, and deceased cases (Tomar and Gupta 2020). The prediction of all cases is performed with LSTM with curve fitting. Moreover, the impact of social isolation and lockdown effect is also measured for COVID-19. In another work, authors have provided a mathematical approach for simulating the spread of COVID-19 to achieve disease-free equilibrium for predictions. The computation of the reproduction of transmissibility with local stability is also investigated (Ndairou et al. 2020). The reproduction number refers to the ratio of restriction rate to the recovery rate. The sensitivity of the COVID-19 model was measured for each parameter selected for analyzing the outbreak in Wuhan, China. A dynamic model for COVID-19 is provided in Cao et al. (2020) for predicting the pattern of infection spread on the population of China. The time series and kinetic model is applied for analyzing the virus spread by considering the preventive and control measures taken in China. The infection rate, mean latency, and fatality rates were taken as input parameters to perform spread prediction. The study showed a decline in the rate of infection and reproduction number. Also, it was found that the increase in recovery rates remained insensitive to the cumulative count of total confirmed cases. Another research on the severity of the disease is examined agewise from the transmission dynamics of coronavirus $(\mathrm{Wu}$ et al. 2020). The criticality of spread is measured in terms of infection fatality risk and symptomatic case fatality risk. The susceptible-infected-recovered model was used for simulation endemic in Wuhan. The authors found that victims of age below 30 and above 59 were having a 5.1 times higher probability of death due to corona. In the previous research, a novel methodology was designed for discovering influencing nodes in an interconnected network (Jain and Sinha 2020). This weighted correlation approach was applied on a real-time social media trend \#CoronavirusPandemic to find highly influencing profiles who are creating awareness on information related to COVID-19, for instance, WHO, health ministries, etc. This can help people to identify authenticated knowledge sources on the web, especially during global health emergencies. There are several risk factors associated with the increased mortality rate of the deadly virus. An extended study relative to coronavirus was conducted to reveal the patients having poor prognosis at the initial stage (Zhou et al. 2020). The 
research initiative proposed in Park et al. (2020) examines the impact of asymptomatic features on the spreading potential of coronavirus for further estimating the regeneration number. The authors were able to estimate the relative case duration of asymptomatic transmissions. In another contemporary work, the impact of restricting travels and quarantine measures was extensively studied on the spread of the pandemic (Hossain et al. 2020). The dynamics of the disease outbreak was analyzed by determining the probability of community spread. The authors found that with border control and isolated treatment strategy could reduce the reproduction number from 2.92 to 1.4 , i.e. if a lockdown procedure is at least as stringent that it can restrict $50 \%$ of the movement, then this can lead to a controlled disease spread scenario. Hence, restriction rate can be assumed to be 0.5. Apart from the physiological factors of patients, recent studies show how the presence of certain environmental pollutants can exaggerate the corona virus symptoms and therefore, contribute as one of the major factors for expediating the spread of the pandemic (Bontempi et al. 2020; Gujral and Sinha 2021).

The current research focussed on analyzing the reduction in the trend of COVID-19 cases in India, particularly during the five phases of lockdown. A network diffusion system is developed that predicted the number of days required to flatten the peak of infected cases in India, based on the then scenario of the virus spread in India. However, presently the withdrawal of lockdown has led to an increase in the number of coronavirus casualties, thereby leading to delays in the cure. The experimental results endorse the reinforcement of lockdown extension as a significant measure to combat the pandemic and bring down the coronavirus to reprieve. The following section shows derivation of the proposed Phase-wise SIR (PSIR) diffusion to model the coronavirus infection rate in India.

\section{Mathematical framework: proposed psir model}

This section highlights the mathematical formulation of proposed PSIR diffusion model. The proposed model considers the lockdown period being stratified into phases, $i=5$ with the beginning and ending tenures being marked by $b_{i}$ and $e_{i}$ respectively. The relative rate of susceptibility to the infection, $\alpha_{i}$ is given by the following Eq. (1), $\forall i$.

$\alpha_{i}(t)_{\left.\right|_{t=e_{i}}}=\max \sum_{x=b_{1}}^{e_{i}} \frac{s_{x}-s_{x-1}}{s_{x-1}}$

The recovery rate for phase $i$ is denoted by $\beta_{i}$ and is computed $\forall i$ from the following equation. $\beta_{i}\left(t, d_{i}\right)_{\left.\right|_{t=e_{i}}}=\frac{1}{d_{i}} \sum_{x=b_{1}}^{e_{i}} \frac{r_{x}}{s_{x}}$

In the above Eq. (2), $d_{i}$ represents the number of days cumulated till phase $i$ and can be expressed as, $d_{i}=e_{i}-b_{1}$. A Phase-wise Susceptible-Infected-Recovered $(P S I R)$ diffusion system is proposed for simulating the impact of lockdown phases on the rate of spread of COVID-19 in India. The number of susceptible and recovered people in lockdown phase $i$ till time $e_{i}$ are characterized by $S_{i}(t)_{\mid t=e_{i}}$ and $R_{i}(t)_{\mid t=e_{i}}$ respectively. It is assumed that the number of infected populations for phase $i$ is dependent on the maximum rate of infection for analyzing the worst-case behavior of the spreading intensity of the virus. The SIR model formulations $\forall i$ are as follows.

$\frac{d S_{i}}{d t}=-\alpha_{i}(t)_{\left.\right|_{t=e_{i}}} S_{i}(t) I_{i}(t)$

$\frac{d I_{i}}{d t}=\left[\alpha_{i}(t)_{\left.\right|_{t=e_{i}}} S(t) I(t)\right]-\left[\beta_{i}\left(t, d_{i}\right)_{\left.\right|_{t=e_{i}}} I_{i}(t)\right]$

$\frac{d R_{i}}{d t}=\beta_{i}\left(t, d_{i}\right)_{\left.\right|_{t=e_{i}}} I_{i}(t)$

In the above phase-wise lockdown analysis, the initial conditions chosen for computing the diffusion rate in proposed PSIR includes the initial susceptible population, $s_{0}=P_{I n}$, where $P_{I n}$ refers to the total population of India dated 15-June-2020. Also, the initial infected population is computed from $v_{0}=\pi_{\infty} \bar{\alpha}$, where $\pi_{\infty}$ refers to the total active cases before phase 1 of lockdown was imposed. From the dataset, $\pi_{\infty} \sim 216$ that is recorded for $d_{\pi}=63$ days starting from 22nd January to 24th March 2020. Finally, the initial condition for the recovered population, $r_{0}=0$. Now, on solving Eqs. (3) and (5), the following expression for $S_{i}(t)$ is derived.

$$
\begin{aligned}
& \frac{d S_{i}}{d R_{i}}=\frac{\alpha_{i}(t)_{\left.\right|_{t=e_{i}}}}{\beta_{i}\left(t, d_{i}\right)_{\left.\right|_{t=e_{i}}}} \Rightarrow \int d S_{i}=\int \frac{\alpha_{i}(t)_{\left.\right|_{t=e_{i}}}}{\beta_{i}\left(t, d_{i}\right)_{\left.\right|_{t=e_{i}}}} d R_{i} \\
& \Rightarrow \ln S_{i}(t)=e^{\frac{-\alpha_{i}(t)_{t=e_{i}}}{\bar{\beta}_{i}\left(t,\left.d_{i}\right|_{t=e_{i}}\right.} R_{i}(t)}+C_{1}
\end{aligned}
$$

Putting the initial conditions in Eq. (6), the following relation is obtained.

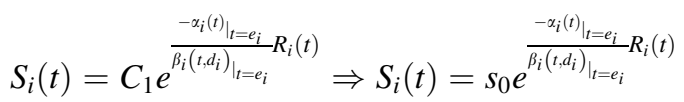

On equating the expressions in (3) and (4), the following expression is derived for the infected population in terms of susceptible and recovered population, using the initial conditions from lockdown phases. 


$$
\begin{aligned}
\frac{d I_{i}(t)}{d S_{i}(t)} & =\frac{\alpha_{i}(t)_{\left.\right|_{t=e_{i}}} S_{i}(t) I_{i}(t)-\beta_{i}\left(t, d_{i}\right)_{\left.\right|_{t=e_{i}}} I_{i}(t)}{-\alpha_{i}(t)_{\mid=e_{i}} S_{i}(t) I_{i}(t)}=-1+\frac{\beta_{i}\left(t, d_{i}\right)_{\left.\right|_{t=e_{i}}}}{\alpha_{i}(t)_{\left.\right|_{t=e_{i}}} S_{i}(t)} \\
& \Rightarrow \int d I_{i}(t)=\int\left(-1+\frac{\beta_{i}\left(t, d_{i}\right)_{\left.\right|_{t=e_{i}}}}{\alpha_{i}(t)_{\left.\right|_{t=e_{i}}} S_{i}(t)}\right) d S_{i}(t) \\
& \Rightarrow I_{i}(t)+S_{i}(t)=\frac{\beta_{i}\left(t, d_{i}\right)_{\left.\right|_{t=e_{i}}}}{\alpha_{i}(t)_{\left.\right|_{t=e_{i}}}} \ln S_{i}(t)+C_{2}
\end{aligned}
$$

Applying the initial conditions in Eq. (8), the value of the integration constant $C_{2}$ can be derived as follows.

$C_{2}=v_{0}+s_{0}-\frac{\beta_{i}\left(t, d_{i}\right)_{\left.\right|_{t=e_{i}}}}{\alpha_{i}(t)_{\left.\right|_{t=e_{i}}}} \ln s_{0}$

Plugging the value of $C_{2}$ from Eq. (9) and $S_{i}(t)$ from Eq. (7) into Eq. (8), the expression for the infected population becomes:

$$
\begin{aligned}
& I_{i}(t)+S_{i}(t)-\frac{\beta_{i}\left(t, d_{i}\right)_{\mid t=e_{i}}}{\alpha_{i}(t)_{\mid t=e_{i}}} \ln S_{i}(t)=v_{0}+s_{0}-\frac{\beta_{i}\left(t, d_{i}\right)_{\mid t=e_{i}}}{\alpha_{i}(t)_{\mid t=e_{i}}} \ln s_{0} \\
& \Rightarrow I_{i}(t)+S_{i}(t)=v_{0}+s_{0}-\frac{\beta_{i}\left(t, d_{i}\right)_{\left.\right|_{t=e_{i}}}}{\alpha_{i}(t)_{\left.\right|_{t=e_{i}}}} \ln \left(\frac{S_{i}(t)}{s_{0}}\right)
\end{aligned}
$$

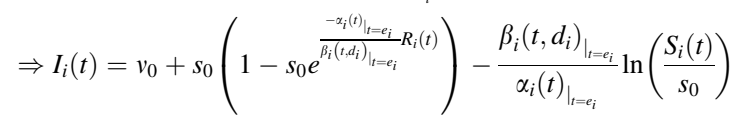

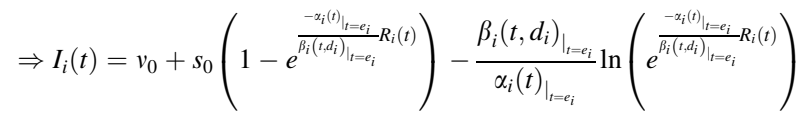

By applying rule: $\ln e^{a}=a$, to the Eq. (10), the expression for the infected population gets further simplified as follows.

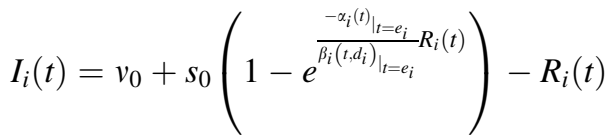

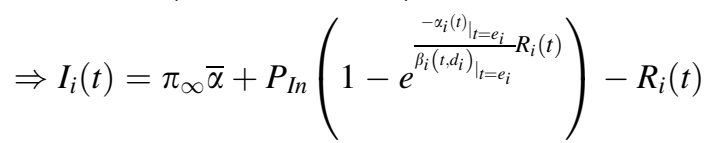

It is known that $s_{0}=P_{I n}$ and $v_{0}=\pi_{\infty} \bar{\alpha}$. The proposed PSIR is finally used to model the phase-wise instantaneous infected population $I_{i}(t)$ in the following section to examine the rate of diffusion of coronavirus spread in India, along with predicting the number of days required to flatten the pandemic peak.

\section{Experimentation outcomes \& analysis}

In the lockdown analysis, the level of infection rate in India was considered an important factor for assessing the impact of the deadly virus. Hence, analyzing the speed of recovery and the rate of infection forms the basis of the research. The reproduction number, $R_{0}$ is defined as the ratio of restriction rate to the recovery rate and is considered one of the standard metric to identify the intensity of virus spread. The restriction rate represents the stringency of lockdown, and recovery rate, being an instantaneous parameter, is expressed as the fraction of total cases recovered to the active cases being detected. Lower is the rate of virus reproduction, faster becomes the rate to flatten the pandemic curve. This section highlights the resultant outcomes from the implementation of proposed PSIR model using Python programming language. The important Python libraries used for modeling and analysis include pandas, numpy, math, scipy.integrate, and scipy.interpolate. Moreover, seaborn and matplotlib libraries were employed for performing informative data visualization and highlevel statistical graphics.

\subsection{Dataset description}

The cases of coronavirus in India were collected from Kaggle dated 1st August 2020, which sources back to aggregated situation reports released by the WHO (COVID-19 Dataset. 2020; World Health Organization 2020). The dataset was chosen for the research due to following reasons:

a. Authentic data source The availability of the dataset on Kaggle that claimed to have real, verified and authentic instances from the world-wide standard organization, i.e. WHO. Moreover, the dataset was updated against the reports received on daily basis from across the globe.

b. Holistic framework The India COVID-19 data was exhaustively framed, with cumulative as well as new instances of active and recovered cases, along with death casualties. This greatly assisted in preparing the parameters for proposed PSIR diffusion model.

The proposed approach highlights the analytical study required for evaluating the pandemic curve for 98 days beginning from 25th March till 30th June 2020. Moreover, extension to the lockdown analysis is also added with case study of the unlocking phase of another 153 days. 


\subsection{Phase-wise lockdown analysis}

The emergency intervention strategies and preventive measures imposed in the early stage had restrained the pandemic to attain its potential. The first phase of lockdown was a significant precautionary agenda to control the deadly contagion. Figure 1a-e shows the increase in recovered cases across the period of lockdown, which eventually got merged with the active cases. This shows the speed in recovery due to the controlled growth in the number of active cases, which became feasible owing to the restricted mobility and strict social distancing norms among people, especially in public places and private localities.

Moreover, due to the comparatively lesser number of daily cases, the availability of medical services to existing confirmed cases became feasible, thus reducing the death casualties. The no-lockdown duration $\left(d_{\pi}\right)$ of the initial 63 is not included in the lockdown analysis. The rate of recovered cases concerning the number of active corona patients rises from phases 1 to 5, as shown in Fig. 2a-e.

Early implementation of lockdown strategies in India resulted in a mild rise in the death rate from 3.35 to $5.13 \%$ through the first to fifth phase (Fig. 3a-d). The COVID

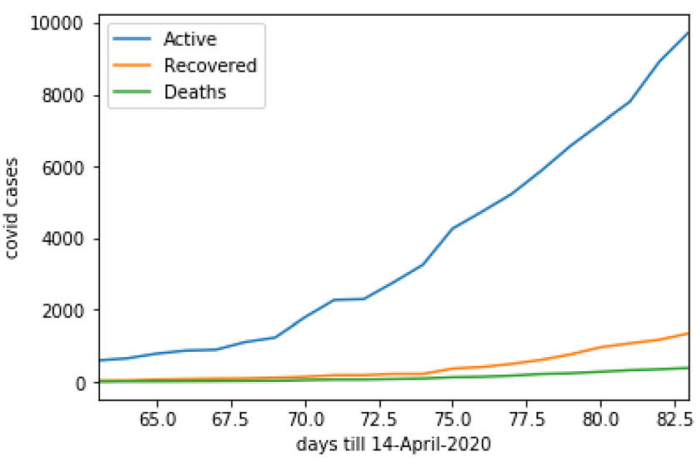

(a)Phase-1

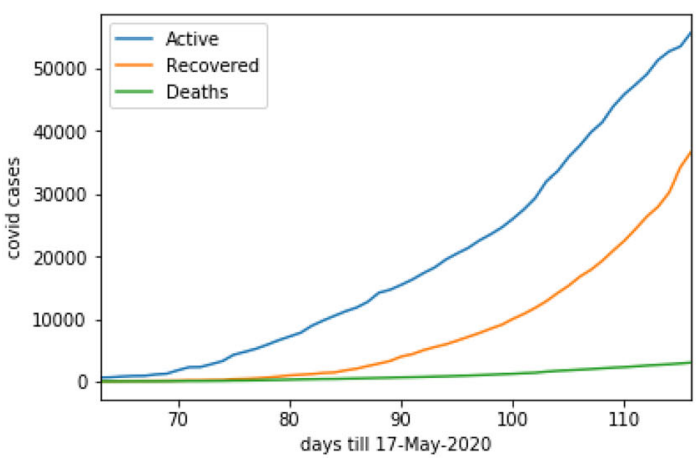

(c) Phase-3

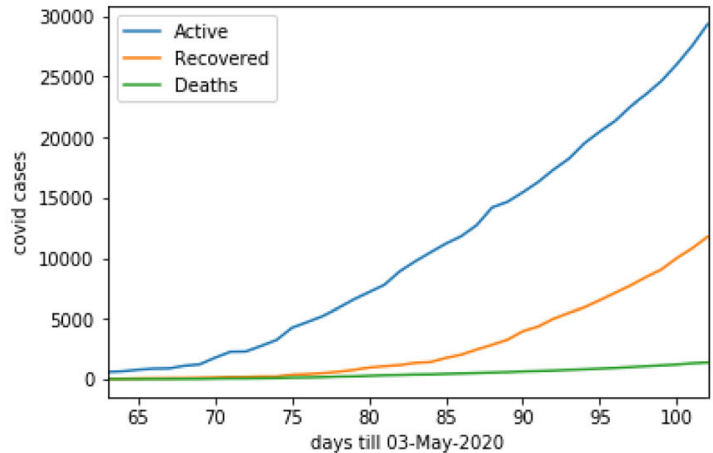

(b) Phase-2

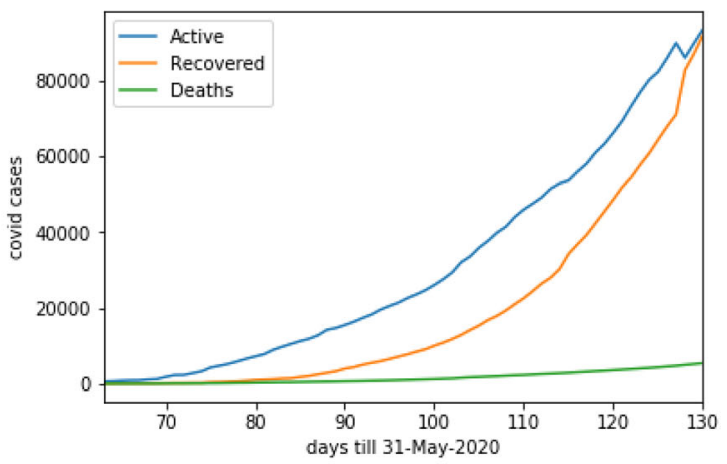

(d) Phase-4

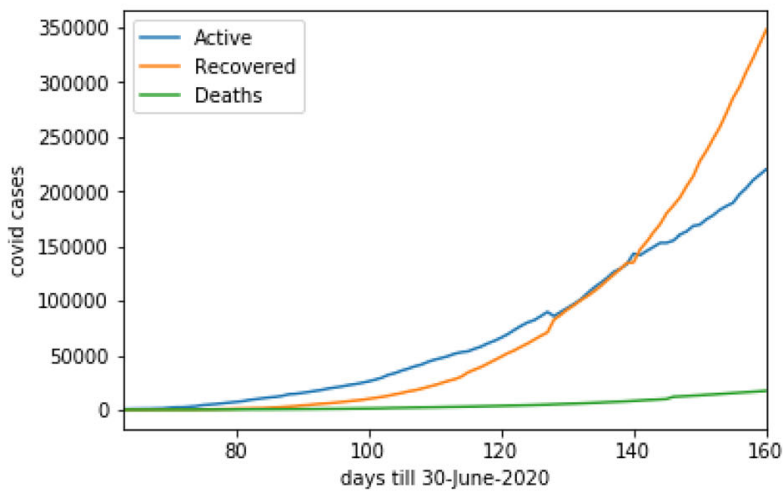

(e) Phase-5

Fig. 1 Number of active, recovered, and death casualties of coronavirus during each lockdown phase in India 


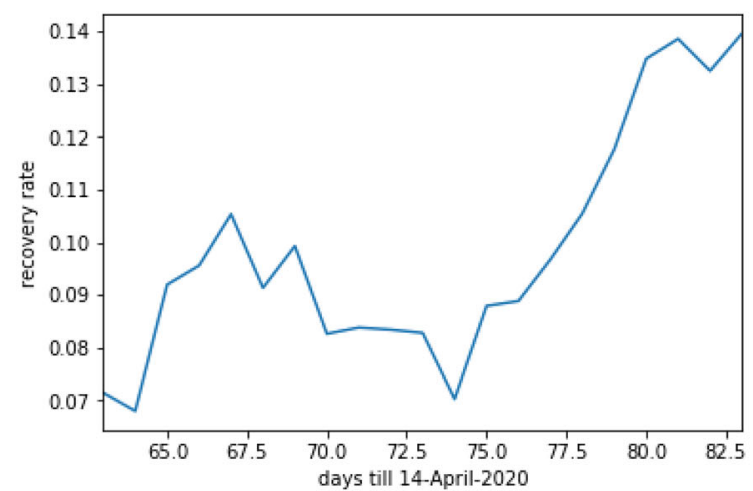

(a) Phase-1

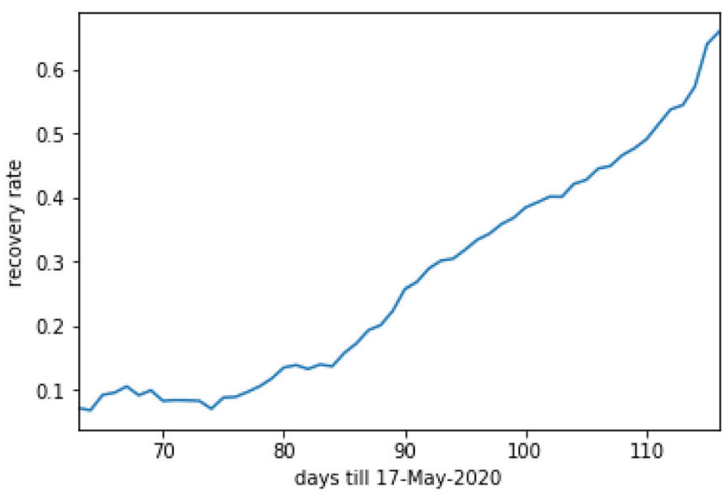

(c) Phase-3

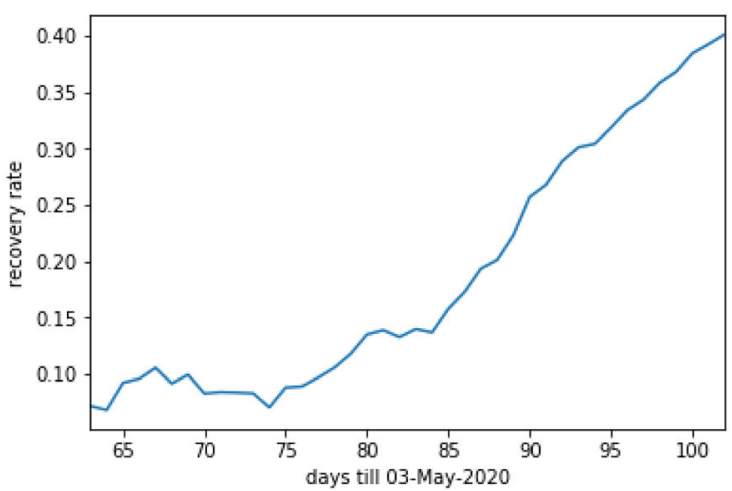

(b) Phase-2

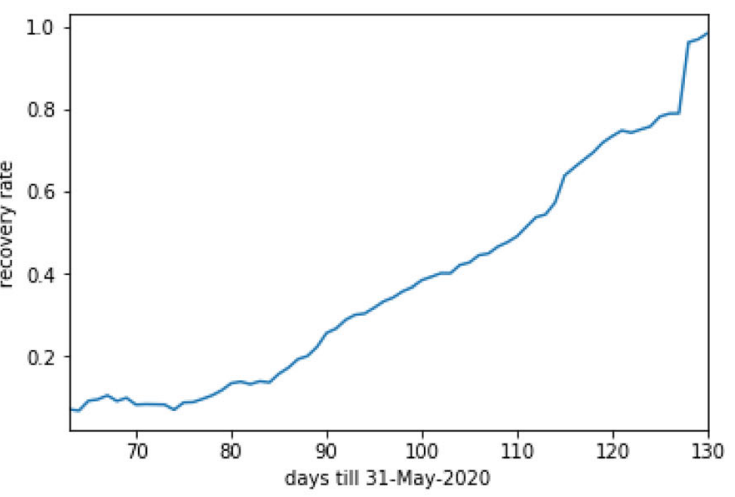

(d) Phase-4

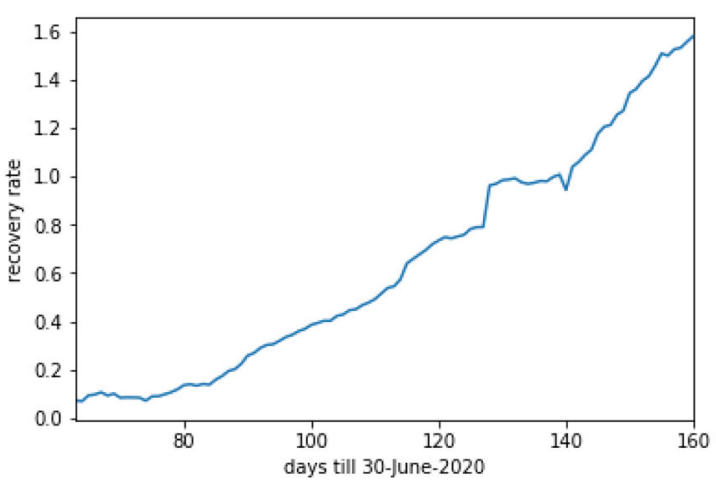

(e) Phase-5

Fig. 2 Phase-wise recovery-rate

research across the country has evidence of a relatively lower death rate as compared to recovered ones. Hence, apart from recovery and death statistics, the critical examination of the infection rate is significantly demanding. The results illustrated in Fig. 4(a-e) show the considerable drop in the rate of infection $\left(\alpha_{i}\right)$ with the maximum value being recorded is 0.4463 . However, by the end of the final phase of lockdown, the mean infection rate dropped to 0.0650 from an initial mean value of 0.1565 .

In Fig. 5, the behavior of deceased cases is analyzed further for measuring the severity of corona in India. For this purpose, two parameters are used that include death-toactive ratio and death-to-recovery ratio. From phase-1 onwards, the death-to-active ratio provides evidence of more active cases concerning deaths. However, this ratio has increased throughout lockdown showing concern for the probability of a future increase in death rate. Hence, more preventive and early remedial measures are required for critical cases. The death-to-recovery ratio is found very high during phase-1 of lockdown. However, this ratio considerably improves towards the end of the 5th phase. 


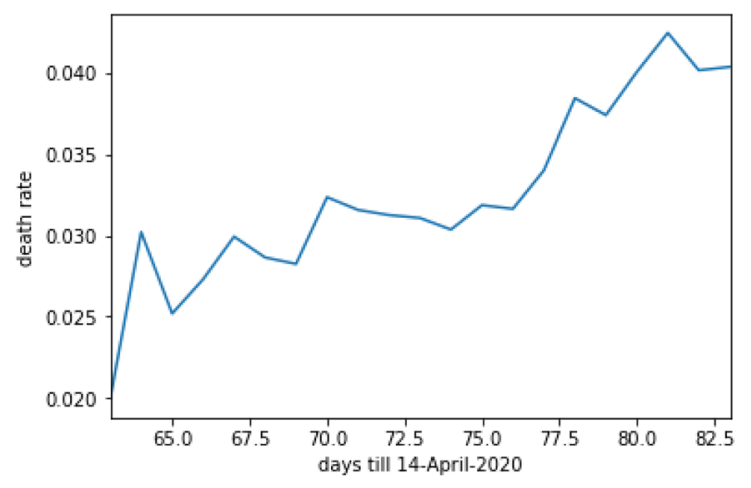

(a) Phase-1

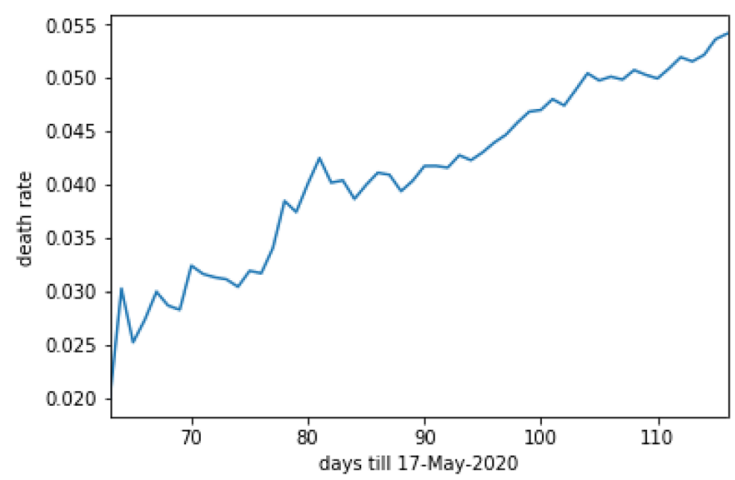

(c) Phase-3

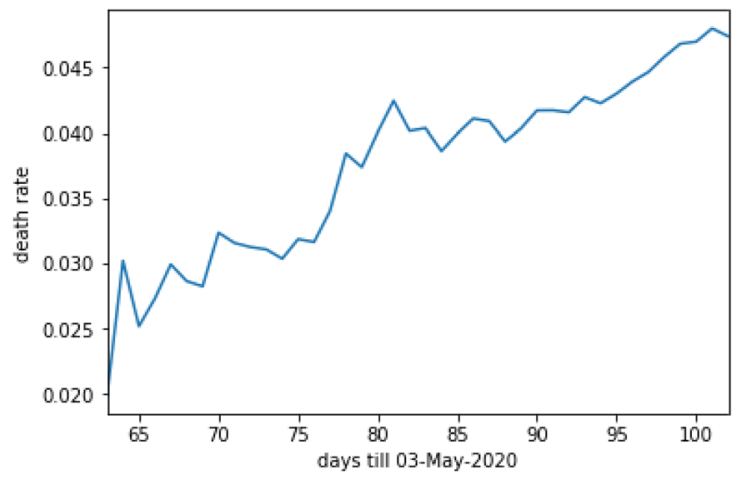

(b) Phase-2

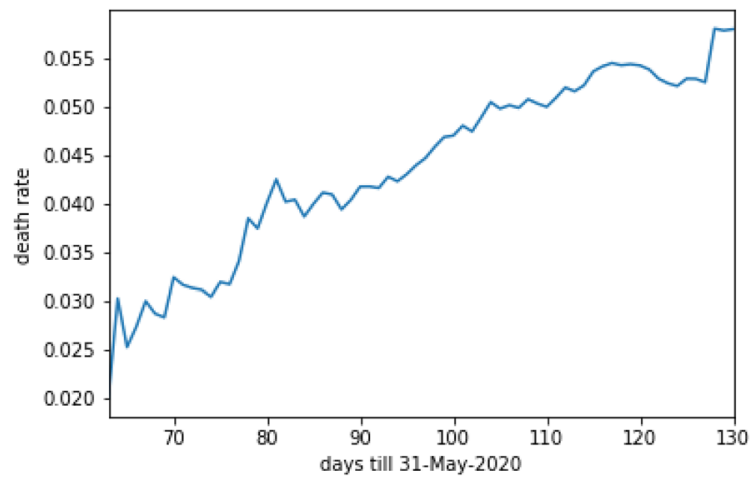

(d) Phase-4

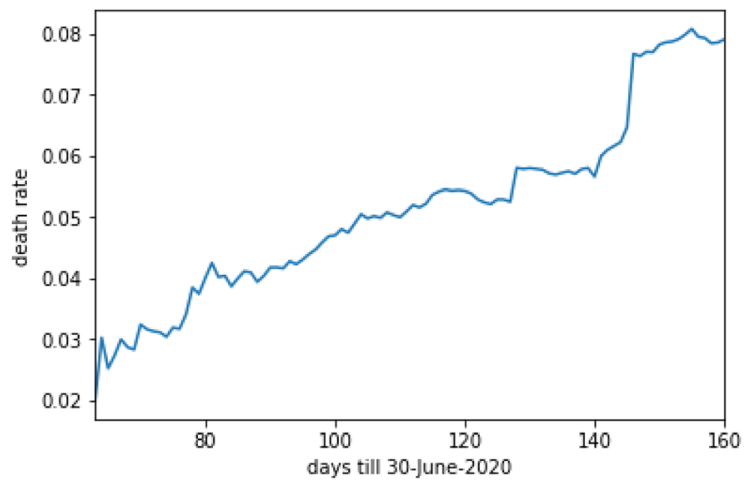

(e) Phase-5

Fig. 3 Phase-wise death rate

The connection between daily deaths with respect to the correlation between active and the recovered cases in India is quite significant. It can be concluded that the increase in the recovery rate affects the minimized deaths in India. It is observed that by the end of phase- 5 lockdown, the ratio between daily cases of deaths and recovery is 0.1436 ; while the ratio concerning daily cases of COVID positives and recovery is 0.1 .4279 (Fig. 6). This indicates a considerable improvement in the recovering instances. The epidemiological parameters obtained from the phase-wise lockdown analysis are highlighted in Table 1.

\subsection{Prediction on flattening the pandemic curve}

The diffusion of COVID-19 in India is simulated using the proposed Phase-wise SIR (PSIR) model and the infected population estimation, $I_{i}(t)$ derived in Eq. (11). The flattening of the pandemic curve is visible in Fig. 7 from phases 1 to 5 . With cluster-containment strategy during the 


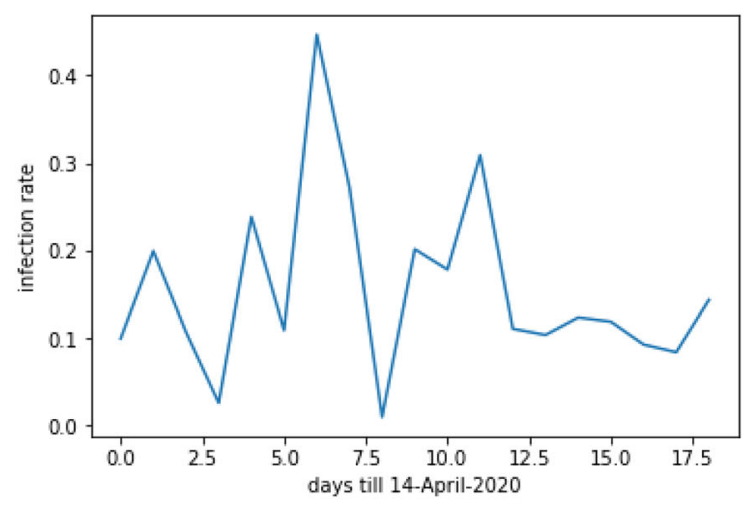

(a) Phase-1

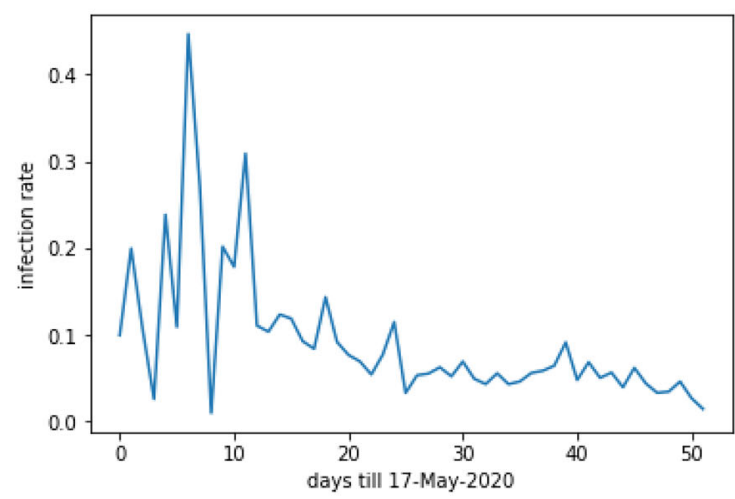

(c) Phase-3

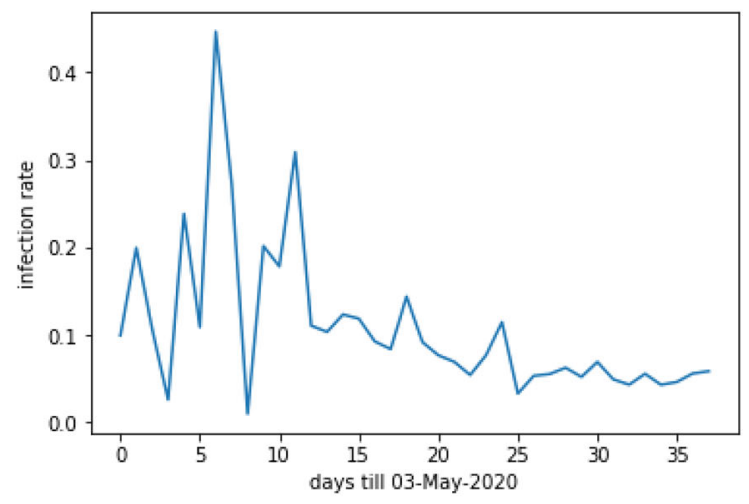

(b) Phase-2

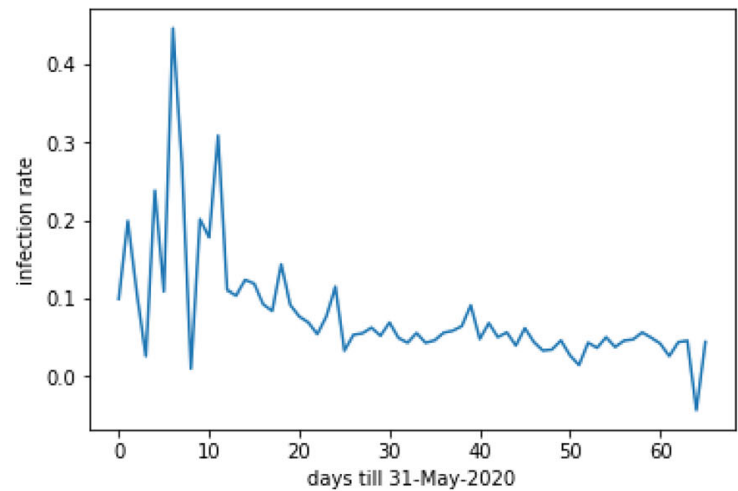

(d) Phase-4

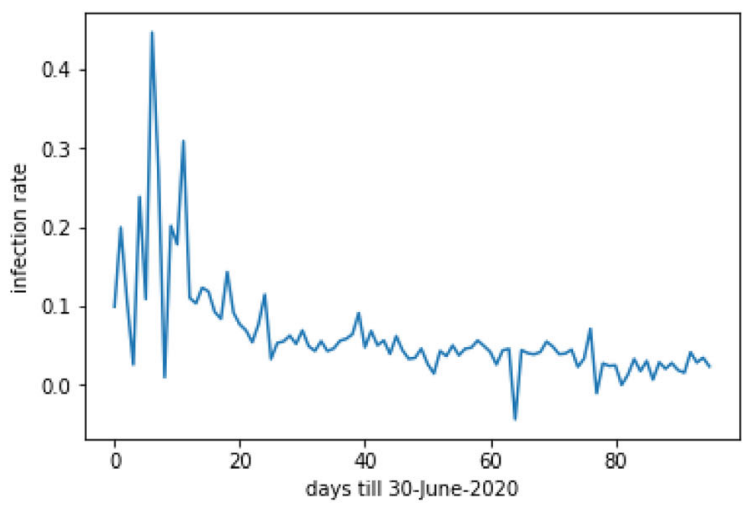

(e) Phase-5

Fig. 4 Phase-wise infection rate

lockdown period helped in early detection and isolation of corona suspected cases. Several active cases were quarantined with the process of contact-tracing of individuals who possibly traversed through corona-affected regions.

In the last phase of lockdown, the infection curve completely flattens as the reproduction number drops to 0.7054 (Fig. 7e). Figures 8a-e show the post-phase prediction of the duration required to minimize the number of infected cases. The number of days by the end in which infection cases drop to zero indicates flattening of the pandemic curve. Further, the actual number of days is highlighted in Table 2.

Table 2 analyses the disease spread intensity based on the reproduction number of coronavirus. It is an instantaneous parameter and is defined as ratio of the rates of restriction and recovery. The rate of restricted mobility is 


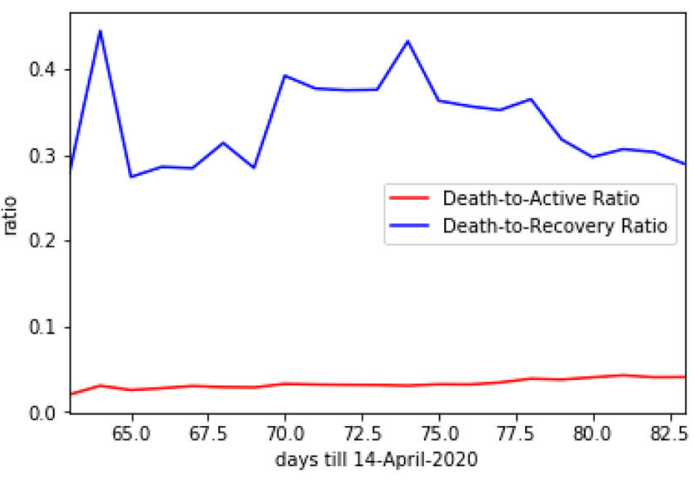

(a) Phase-1

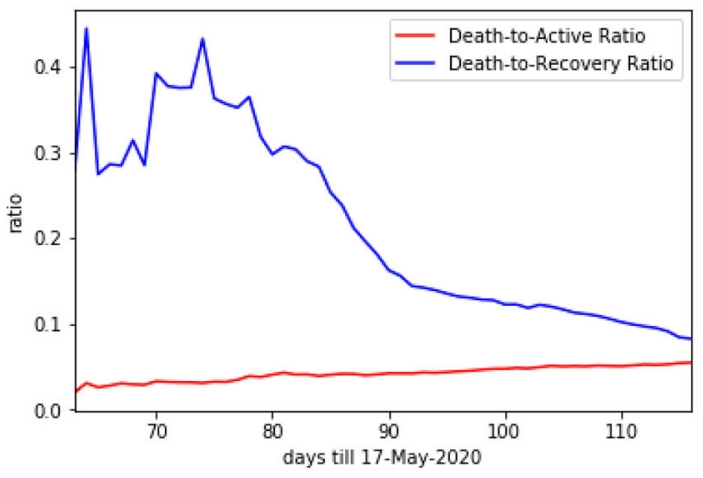

(c) Phase-3

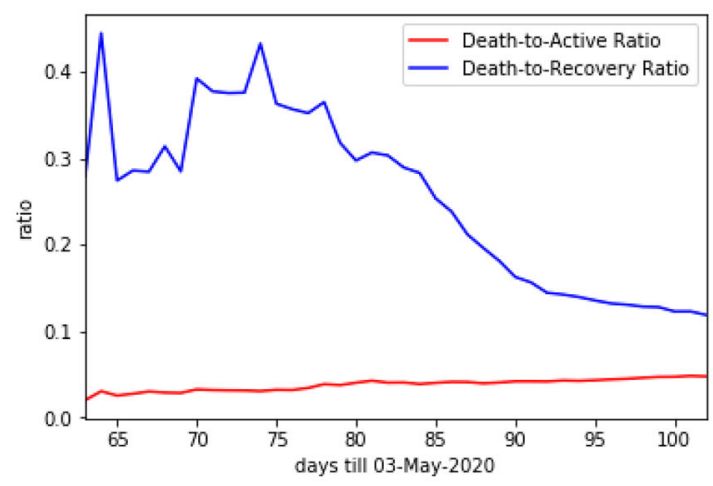

(b) Phase-2

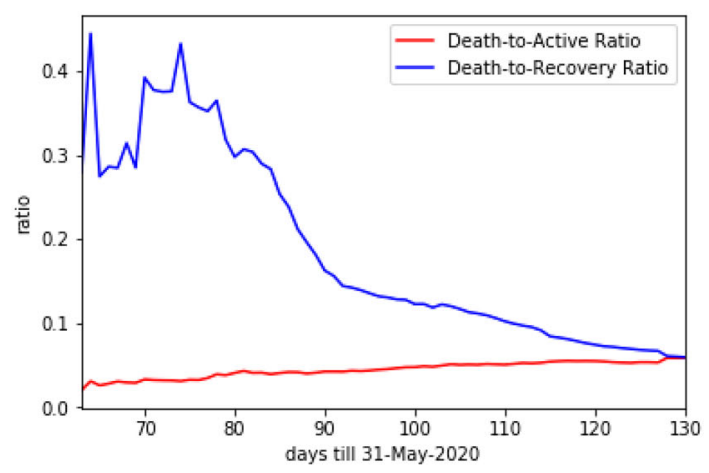

(d) Phase-4

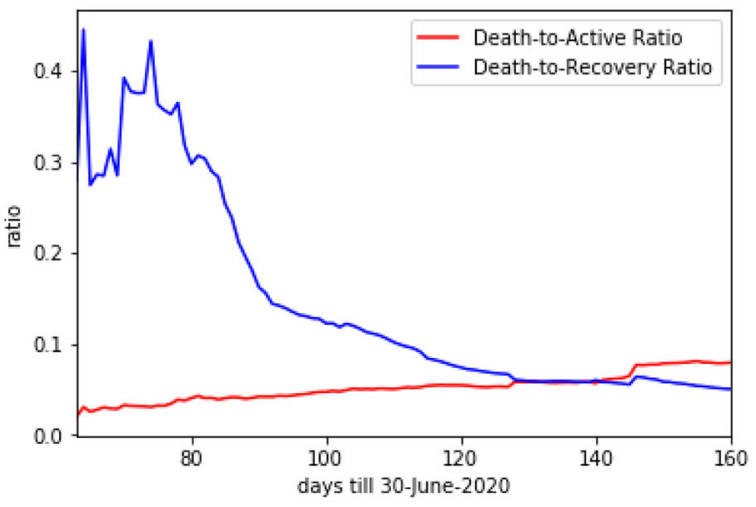

(e) Phase-5

Fig. 5 Phase-wise survival ratio of recovered \& active corona cases

assumed to be $50 \%$ as used in the existing literature, and hence is set to 0.5 . The permissible movement included doctors, nurses, ambulance drivers, medicine shopkeepers, security officials, and other delivery personnel involved in serving to the daily essential needs of the people. From the tabular data, the reproduction number $\left(R_{0}\right)$ of coronavirus in India dropped from 4.5 to 0.7 after five phases of lockdown. However, after the $5^{\text {th }}$ lockdown, became largely devastating with the COVID-19 blast leading to around 9.5 times increase in the number of coronavirus infected patients, as compared to lockdown times. Till the fifth phase of lockdown, 0.566 million cases were reported, which increased to 5.65 million in India (Statistics and Research 2020). The smaller towns and rural areas are also badly hit by the pandemic, along with the megacities, Delhi and Mumbai. The sole reason was the withdrawal of lockdown causing relaxation in the strictness of norms of social distancing and sanitization against coronavirus. Furthermore, the movement of migrant workers, re-opening of shopping malls, and business firms led to the explosion of COVID-19 cases.

The rate of covid reproduction number begun to escalate from the initial phase of unlocking. This eventually led to increase in time taken to curb the infection with a sudden 


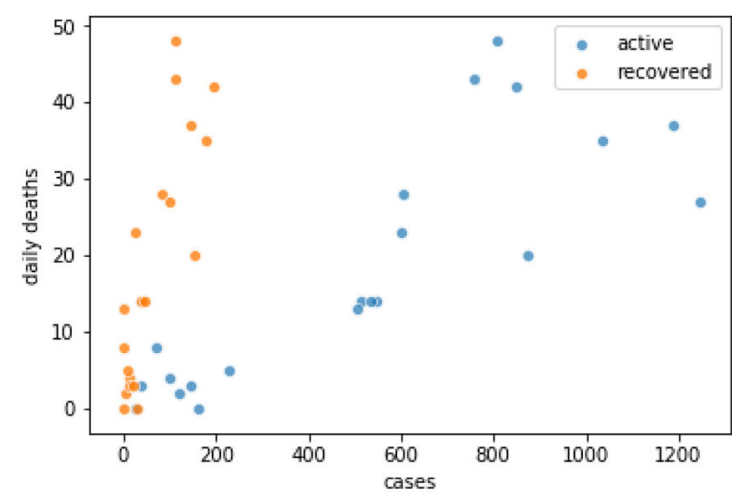

(a) Phase-1

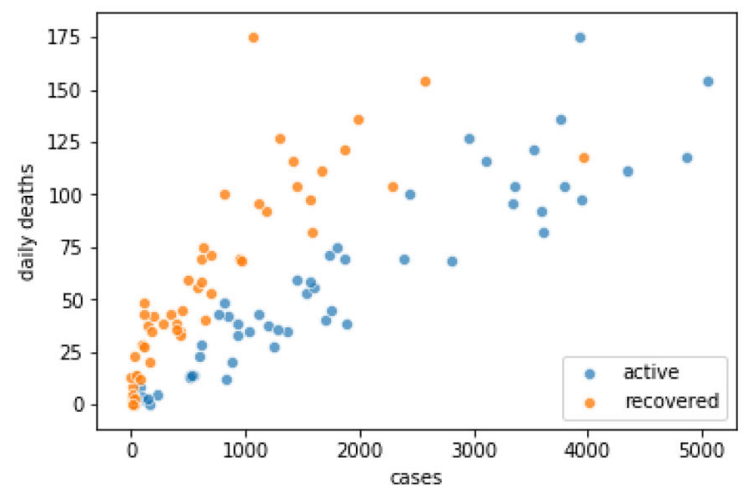

(c) Phase-3

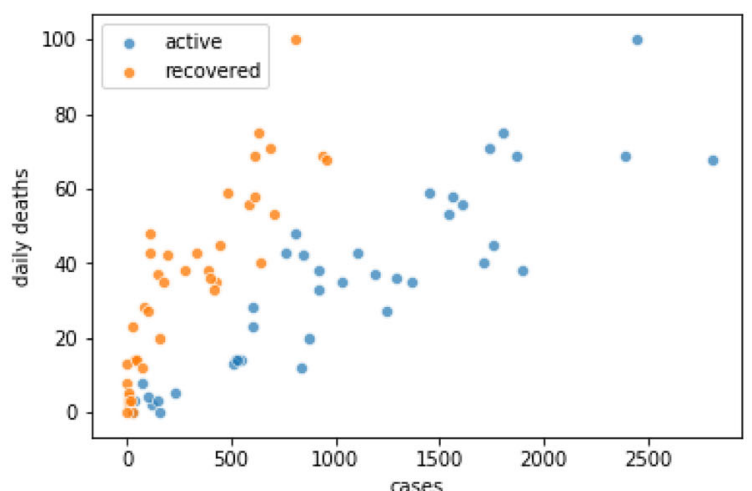

(b) Phase-2

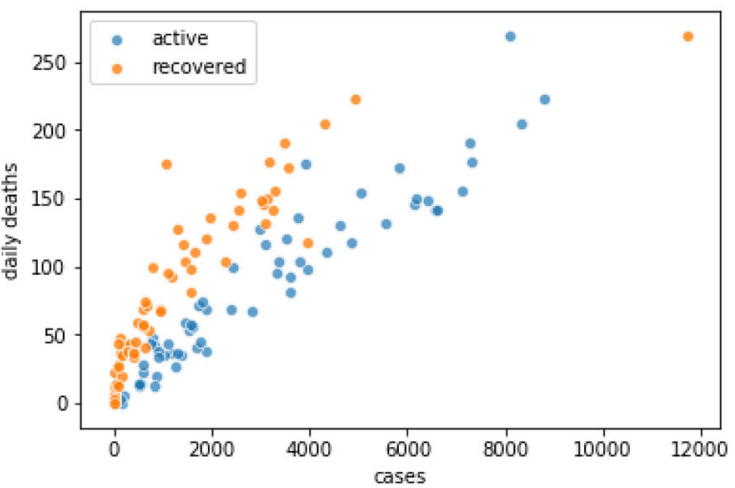

(d) Phase-4

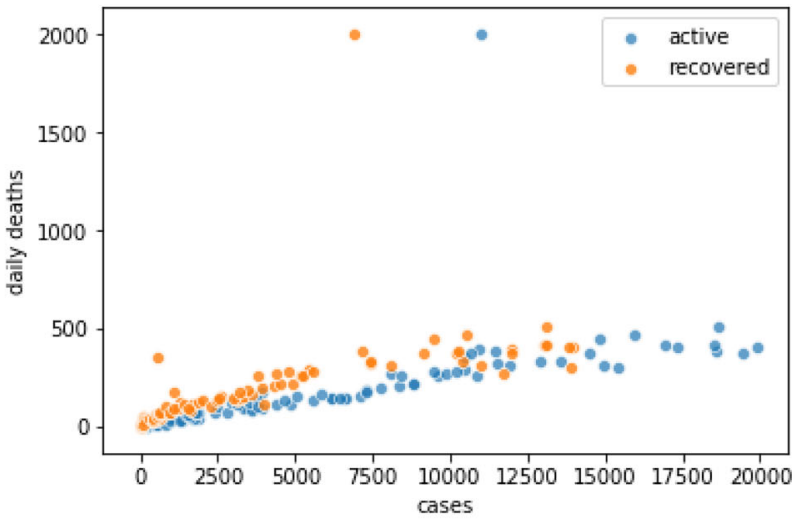

(e) Phase-5

Fig. 6 Study of the deceased cases concerning everyday active and recovered cases of COVID-19

Table 1 Epidemiological parameters for coronavirus in lockdown

\begin{tabular}{lllll}
\hline Lockdown phase & Lockdown phase tenure & Mean infection rate & Mean recovery rate & Mean death rate \\
\hline 1 & 25th March-14th April & 0.1565 & 0.0984 & 0.0325 \\
2 & 15th April-3rd May & 0.1089 & 0.1867 & 0.0374 \\
3 & 4-17th May & 0.0927 & 0.2688 & 0.0409 \\
4 & 18-31st May & 0.0811 & 0.3767 & 0.0437 \\
5 & 1-30th June & 0.0650 & 0.6326 & 0.0513 \\
\hline
\end{tabular}




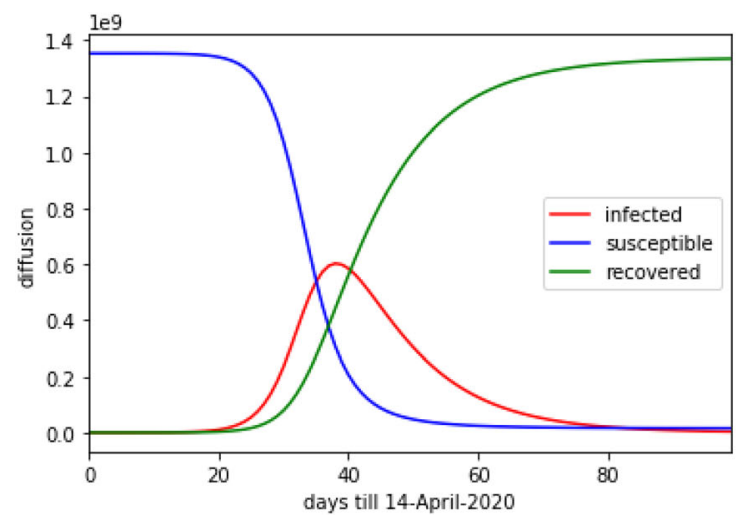

(a) Phase-1

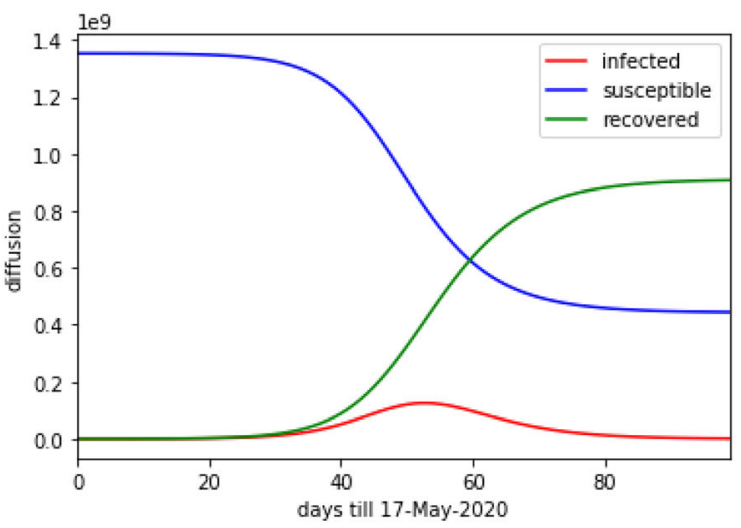

(c) Phase-3

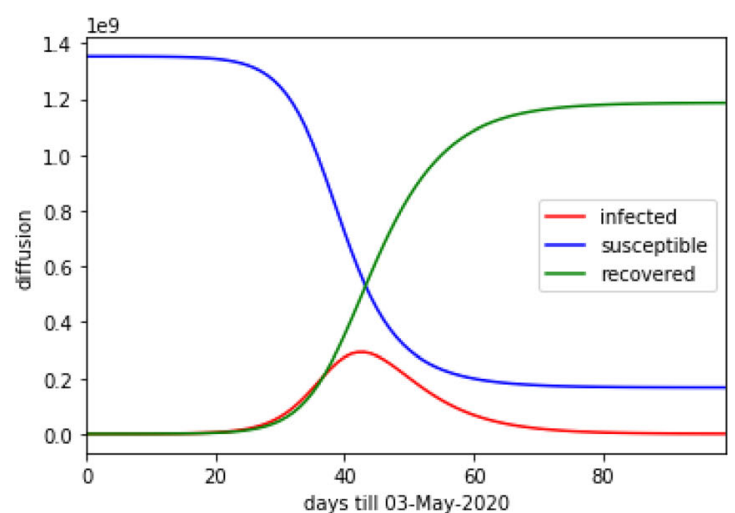

(b) Phase-2

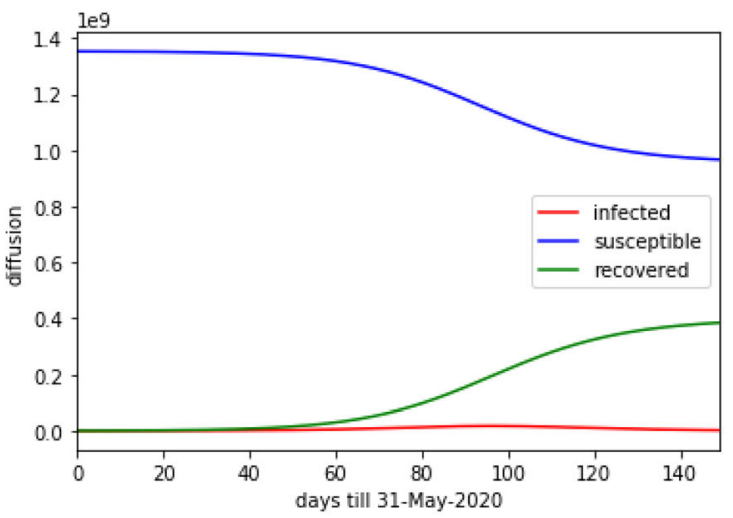

(d) Phase-4

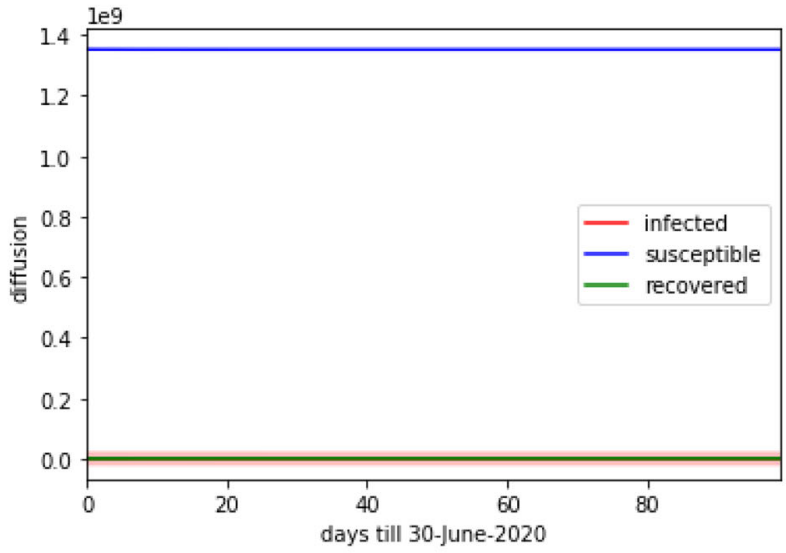

(e) Phase-5

Fig. 7 Diffusion of coronavirus over the population of India by the end of lockdown phases

rise in the susceptible population, thereby introducing more delays in the cure. Moreover, Fig. 9 shows the evolving trend of the virus reproduction number, $R_{0}$ for lockdown and unlocking phases.

It is apparent from the graph that as the unlocking begun from July 2020, the value of $R_{0}$ also started to rise due to eventual relaxation in social distancing and sanitization measures amongst people. This outcome from proposed
PSIR provides a possible prediction of the second wave, which eventually occurred in India around March 2021. This justifies the utility of the proposed PSIR model. Though, we know that lockdown for a longer time is practically not feasible, hence, one may rely on the rate of rise in the reproduction number of coronavirus to regulate the stringency and duration of lockdown phases for curtailing the viral threat. 


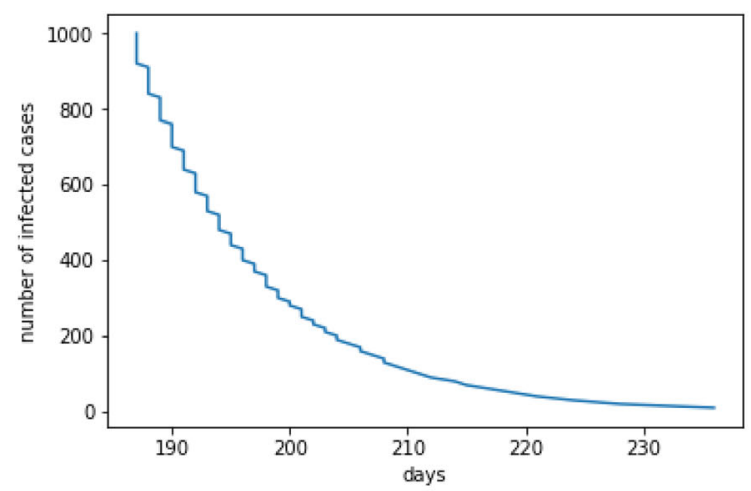

(a) Phase-1

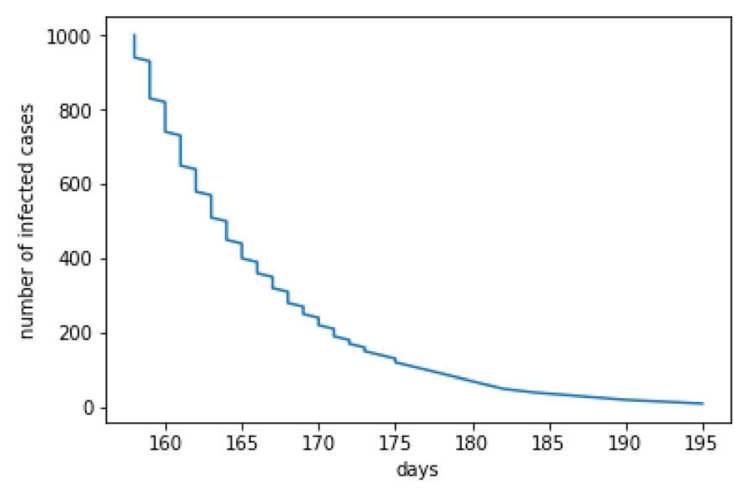

(c) Phase-3

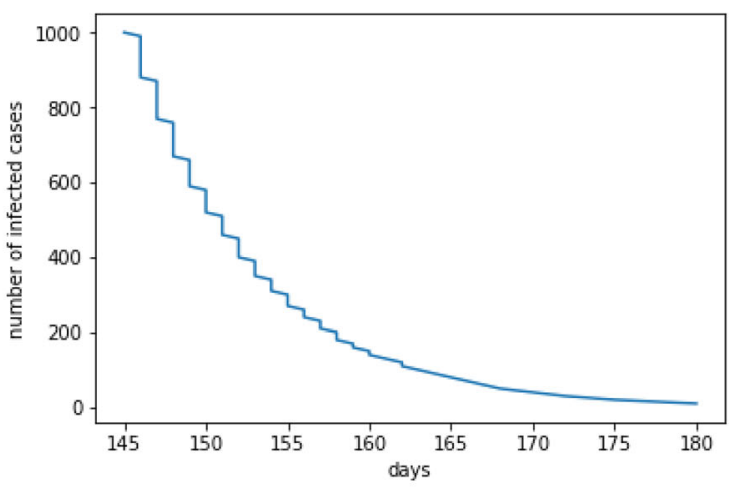

(b) Phase-2

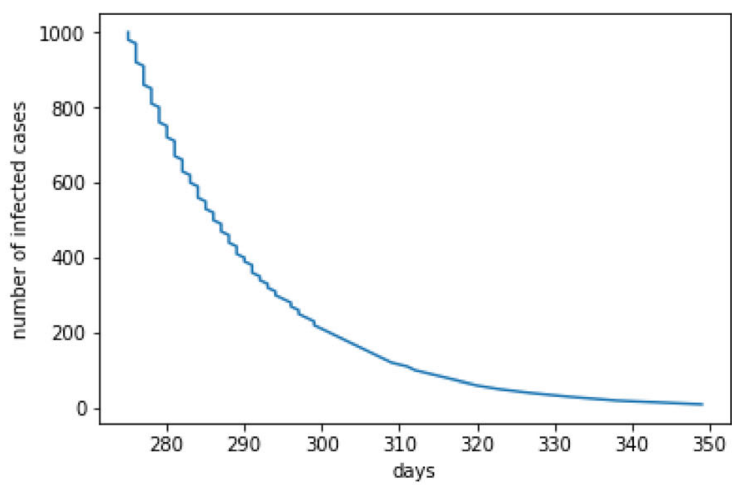

(d) Phase-4

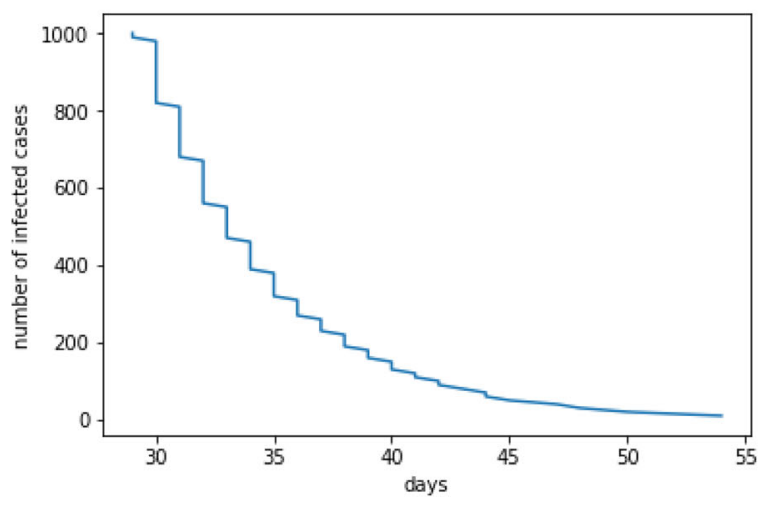

(e) Phase-5

Fig. 8 Prediction of the number of days required for the infected cases to drop to zero

\section{Conclusion}

The lockdown process has great significance in averting the danger of the coronavirus from entering into community spread transition. Initially, the lockdown strategy was imposed in India to control the high casualty count due to COVID-19 by canceling travel, sealing national boundary, limiting the free movement of crowds, along with creating hygiene awareness. The proposed work conducts a case study of India for the assessment of five phases of lockdown. For this purpose, a network diffusion-based system is proposed for simulating the disease spread during the phase-wise lockdown in India. Infection rate and recovery rate were found varying with lockdown phases and, the reproduction number of coronavirus consistently decreased from 4.53 to 1.18 by the end of the final phase. With the maximum rate of infection of 0.44 , a customary improvement was observed in the rate of mean recovered cases from $9.84 \%$ to $37.67 \%$. Moreover, other parameters including death-to-active ratio and death-to-recovery ratio 
Table 2 Experimental outcomes from diffusion analysis of lockdown phases

\begin{tabular}{|c|c|c|c|c|c|}
\hline Phase & Mode & Duration (in days) & Phase tenure $^{\dagger}$ & $R_{0}$ & Flattening of pandemic curve ${ }^{*}$ (month) \\
\hline 1 & \multirow[t]{5}{*}{ Lockdown } & 21 & 25th March-14th April & 4.5 & $236-7.70$ \\
\hline 2 & & 19 & 15th April-3rd May & 2.3 & $180-5.91$ \\
\hline 3 & & 14 & 4-17th May & 1.6 & $195-6.41$ \\
\hline 4 & & 14 & 18-31st May & 1.1 & $349-11.47$ \\
\hline 5 & & 30 & 1-30th June & 0.7 & $54-1.77$ \\
\hline 6 & \multirow[t]{5}{*}{ Unlocking } & 31 & $1-31$ st July & 1.8 & $208-6.83$ \\
\hline 7 & & 31 & 1-31st August & 3.5 & $230-7.56$ \\
\hline 8 & & 30 & 1-30th September & 2.1 & $187-6.14$ \\
\hline 9 & & 31 & 1-30th October & 2.0 & $183-6.01$ \\
\hline 10 & & 30 & 1-30th November & 1.7 & $199-6.54$ \\
\hline
\end{tabular}

${ }^{\dagger}$ Tenure: year 2020

*m Refers to months

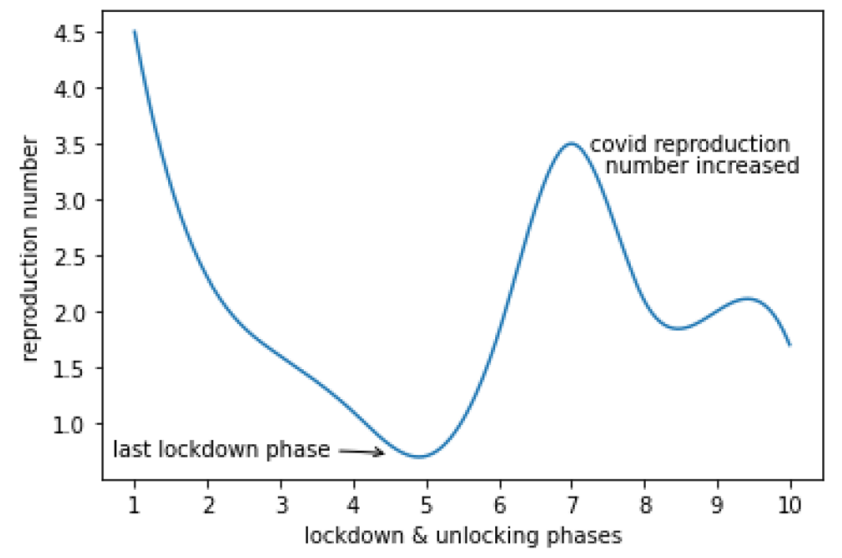

Fig. 9 Trend of coronavirus reproduction number

were incorporated in the analysis to assess the level of immunity against the pandemic. The phase-wise SIR network diffusion model was derived for forecasting the approximate number of days to curb off the coronavirus spread. A steady drop of $23.24 \%$ was observed in the number of days in eliminating the infected cases by the end of the second phase of lockdown. However, relaxation in phase-4 constraints led to an increase in the infection parameter, thus signaling the continuation of another consecutive phase of lockdown. Ultimately, the final lockdown phase with a maximum duration of 30 became successful to flatten the curve of the infected cases with a drastic drop in reproduction number to 0.7054 . However, the withdrawal of lockdown resulted in a huge increase in COVID-19 cases. Hence, based on the study of the rate of rise in the coronavirus reproduction number, it is suggested to regulate the stringency and span of lockdown in India for obviating possibilities of third wave.
Funding No funding was received for conducting research mentioned in this article.

\section{Declarations}

Conflict of interest The author certifies that there is no actual or potential conflict of interest in relation to this article

\section{References}

Bontempi E, Vergalli S, Squazzoni F (2020) Understanding COVID19 diffusion requires an interdisciplinary, multi-dimensional approach. Environ Res 188:109814

Cao J, Jiang X, Zhao B (2020) Mathematical modeling and epidemic prediction of COVID-19 and its significance to epidemic prevention and control measures. J Biomed Res Innov 1(1):1-19

Castelli L, Di Tella M, Benfante A, Romeo A (2020) The spread of COVID-19 in the Italian population: anxiety, depression, and post-traumatic stress symptoms. Can J Psychiatry 65(10):731

COVID-19 Dataset (2020) Number of confirmed, death and recovered cases every day across the globe, Kaggle, https://www.kaggle. com/imdevskp/corona-virus-report

Cucinotta D, Vanelli M (2020) WHO declares COVID-19 a pandemic. Acta Bio-Medica: Atenei Parmensis 91(1):157-160

Gujral H, Sinha A (2021) Association between exposure to airborne pollutants and COVID-19 in Los Angeles, United States with ensemble-based dynamic emission model. Environ Res 194:110704

Gurevich IB, Zhuravlev YI (2014) Computer science: subject, fundamental research problems, methodology, structure, and applied problems. Pattern Recognit Image Anal 24(3):333-346

Hossain M, Junus A, Zhu X, Jia P, Wen TH, Pfeiffer D, Yuan HY (2020) The effects of border control and quarantine measures on global spread of COVID-19. Epidemics 32(2020):100397

Jain S, Sinha A (2020) Identification of influential users on twitter: a novel weighted correlated influence measure for Covid-19. Chaos, Sol Fractals 139:110037

Ndairou F, Area I, Nieto JJ, Torres DF (2020) Mathematical modeling of COVID-19 transmission dynamics with a case study of Wuhan. Chaos, Sol Fractals 135:109846 
Park SW, Cornforth DM, Dushoff J, Weitz JS (2020) The time scale of asymptomatic transmission affects estimates of epidemic potential in the COVID-19 outbreak. Epidemics 31:100392

Sebhatu A, Wennberg K, Arora-Jonsson S, Lindberg SI (2020) Explaining the homogeneous diffusion of COVID-19 nonpharmaceutical interventions across heterogeneous countries. Proc Natl Acad Sci 117(35):21201-21208

Sinha A, Rathi M (2021) COVID-19 prediction using ai analytics for South Korea. Appl Intell. https://doi.org/10.1007/s10489-02102352-z

Statistics and Research, Coronavirus Pandemic (2020), ourworldindata.org/coronavirus

Tomar A, Gupta N (2020) Prediction for the spread of COVID-19 in India and effectiveness of preventive measures. Sci Total Environ 728:138762

Veera Krishna M (2020) Mathematical modelling on diffusion and control of COVID-19. Infect Dis Model J 5:588-597

Vissia H, Krasnoproshin V, Valvachev A (2020) Decision-making technology based on big data. Pattern Recognit Image Anal 30(2):230-236
World Health Organization. (2020). Coronavirus disease situation reports, www.who.int/emergencies/diseases/novel-coronavirus2019/situation-reports

Wu JT, Leung K, Bushman M, Kishore N, Niehus R, de Salazar PM, Leung GM (2020) Estimating clinical severity of COVID-19 from the transmission dynamics in Wuhan China. Nat Med 26(4):506-510

Zemtsov SP, Baburin VL (2020) COVID-19: spatial dynamics and diffusion factors across Russian regions. Reg Res Russia 10(3):273-290

Zhou F, Yu T, Du R, Fan G, Liu Y, Liu Z, Guan L (2020) Clinical course and risk factors for mortality of adult inpatients with COVID-19 in Wuhan, China: a retrospective cohort study. The lancet 395(10229):1054-1062

Publisher's Note Springer Nature remains neutral with regard to jurisdictional claims in published maps and institutional affiliations. 